\title{
Post Activation Potentiation in North American High School Football Players
}

\author{
Granite Tano $^{1}$, Alan Bishop ${ }^{2}$, Joe Berning ${ }^{3}$, Kent J. Adams ${ }^{4}$ and Mark DeBeliso ${ }^{1}$ \\ 1. Department of Physical Education and Human Performance, Southern Utah University, Cedar City, UT 84720, USA \\ 2. Athletics Department, Utah State University, Logan, UT 84322, USA \\ 3. New Mexico State University, Department of Kinesiology and Dance, New Mexico State University, Las Cruces, NM 88003, USA \\ 4. California State University Monterey Bay, Kinesiology Department, Seaside, CA 93955, USA
}

\begin{abstract}
Post activation potentiation (PAP) is an acute enhancement of muscular power production resulting from a high intensity potentiating exercise. Practical applications of the PAP phenomena as a warmup strategy are the focus of ongoing research. This study compared the effects of a dynamic and a PAP warm up strategy on speed and horizontal plane muscular power output. High School male football players $(n=16)$ participated in a repeated measures cross over design study consisting of 3 testing days. Day 1: one repetition maximum (1-RM) back squats were established. Day 2: half of the participants completed a dynamic warmup consisting of dynamic movements (ex. high knees, butt kickers, frog jumps, cherry pickers, lateral slide, karaokes, back pedal) while the other half of the participants performed a PAP warmup consisting of back squats culminating in a set of 4 repetitions at $85 \%$ of 1 -RM. Following the warmups (4-minutes), the participants performed 3 trials of a weighted sled push 91 kilograms (200 pounds) over a 9.1 meter (10 yards) linear path. Following the sled pushes, the participants then performed three trials of 18.2 meter (20 yards) sprints. Day 3: participants crossed over with respect to the warmup procedures and again performed the three sled push and sprint trials. Dependent t-tests were used to compare the sled push and sprint times between warm up conditions. Both the 18.2 meter sprint $(P<0.01)$ and the 91 kilogram sled push $(P<0.01)$ showed a significant improvement when PAP was utilized as a warmup, rather than the dynamic warmup. Within the parameters of the study, it is concluded that PAP as a warm up strategy enhances sprint ability and horizontal plane muscular power output.
\end{abstract}

Key words: PAP, Prowler, football, high school.

\section{Introduction}

Post activation potentiation (PAP) is defined as an acute enhancement of muscle function following an intense muscle activity [1]. The proposed mechanisms leading to the PAP phenomena have been discussed in detail elsewhere [2]. The practical applications of PAP are of growing interest and continue to be explored [2], a practice recommended by Bishop's [3] applied research model for the sport sciences.

Complex training (a form of PAP) has become popular in strength and conditioning programs for athletes. Complex training (CT) is implemented by

Corresponding author: Mark DeBeliso, Ph.D., professor, research fields: orthopedic biomechanics, mechanics and metabolics of sport movements and work-tasks, strength training for all walks of life, and master's athletes. coupling a heavy slower lift (excitation) prior to an explosive exercise or movement within a training session (e.g. a heavy back squat followed by a vertical jump). Sport teams have been interested in the prospect of using CT to accelerate improvements related to strength and conditioning programs and the potential for improving athletic performance. With that said, examining various CT coupling exercises focused more directly on improving athletic performance are of great interest in the sport community

Sprint speed and acceleration are important athletic qualities, which are crucial in a number of team (rugby union, American football, soccer, hockey) and individual sports (sprinting, long jump, skating). The incorporation of sled training as part of strength and power training regimes is now common practice with 
various sports integrating its use for improvements in speed, which is well documented in the literature $[4,5]$.

In addition to speed, many sports require a participant to engage an opponent (or sport implement) and push forward in the horizontal plane. This includes football down linemen, wrestlers, mixed martial arts, rugby athletes, bobsledders, as well as certain Crossfit and strongman events. Sled training, where the athlete pushes a loaded sled in the horizontal plane, closely mimics the horizontal plane power requirements of the aforementioned sports by removing momentum and forcing the athlete to overcome inertia. Sled pushing in the horizontal plane, should be beneficial with respect to transferring muscular strength and power that was developed during traditional strength and conditioning protocols which include squats (back and front) and Olympic lift variations that occur primarily in the vertical plane with simultaneous bilateral leg engagement. Further, sled pushing aids in sport specific motor skill development within a horizontal plane due to total body engagement of the hands, arms, shoulder girdle, trunk, and lower extremity musculature during the push in a closed chain unilateral leg propulsion manner.

Given the training utility, sport specificity, and ease of use, the question arises as to if pairing the sled push with a potentiating (PAP) exercise could lead to an acute improvement to execute the sled push exercise.

Hence, the purpose of this study was to determine if a PAP warmup using a using a progression of back squats (BSQ) sets culminating in a near maximum BSQ is effective in improving sled push performance as opposed to using a dynamic warmup. This study also aimed to determine if the same BSQ PAP warmup strategy could also improve short distance sprint speed.

\section{Methods}

\subsection{Participants}

A convenience sample of High School male football players were recruited and later volunteered for the study $(n=16)$. Permission to conduct the study was obtained through a University Institutional Review Board. Each participant and parent provided written consent prior to engaging in the study.

\subsection{Procedures}

There was three days of testing sessions. On Day 1, all of the participants established a 1-RM BSQ as previously described [6]. The participants used 5-7 progressively loaded sets (greater weight and lower repetitions) separated by 2-3 minutes until they failed the lift or could not maintain proper body mechanics. The 1-RM BSQ score was then used to calculate the load to be used during the PAP warmup session (85\% of 1-RM). The participants were then randomly assigned into two groups.

On Day 2 of testing, half of the participants performed a dynamic warmup and the other half per formed a PAP warmup. After the warmups (4-minutes), each group performed three trials of a weighted sled pushes (9.1 meters or 10 yards) followed by three trials of 18.2 meters (20 yards) sprints. Each trial was separated by 2-3 minutes.

The dynamic warmup strategy consisted of several exercises performed for a distance of 18.2 meters (9.1 meters to and from a marked line). The participants performed one warmup movement moving towards the marked line and switched to another movement on the way back. The movements were: high knees, butt kickers, frog jumps, cherry pickers, lateral slide, karaokes, back pedal, and finished up with a sprint $(\approx 10$ minutes).

The PAP warmup strategy consisted of the participants performing a progression of sets of BSQs with a 3-5 minute rest in-between each set. The weights were predetermined from the 1-RM BSQ recorded during the initial meeting. The warm-up progression was as follows (sets $\times$ repetitions $\times 1$-RM load): $1 \times 5 \times$ $40 \% ; 1 \times 5 \times 60 \% ; 1 \times 5 \times 70 \% ; 1 \times 4 \times 85 \%$. The culminating set at $85 \% 1-\mathrm{RM}$ was considered the potentiating exercise/event.

On Day 3 of testing, the participants crossed-over 
with respect to the warmup strategies and again performed the three trials of a weighted sled pushes followed by three trials of 18.2 meter sprints.

The dependent variables in this study were the weighted sled push and the 9.1 meter sprint. The weighted resistance sled used in this study was the Prowler. The Prowler is a commercially available resistance sled that has two sets of handles that enables the athlete to push the sled horizontally from either a low or high position. Fig. 1 demonstrates an athlete pushing the sled from the high (or vertical) handle position (example picture not exact load).

Each participant performed three maximal effort trials of the Prowler sled push (Fig. 1). The Prowler was loaded with Olympic weights such that the total combined weight was 91 kilograms (200 pounds Prowler plus weights). The loaded Prowler was vertical handle pushed [7] by the participants for a total of 9.1 meters in a direct linear path. The average of the three sprint trials was used for subsequent statistical analysis. The high reliability of the Prowler has been reported with an ICC $=0.93$ [8].

The participants also performed three trials of an 18.2 meter sprint (20 yards) separated by 2-3 minutes. The average of the three sprint trials was used for subsequent statistical analysis. The test-retest reliability of short sprints has been reported to be $r=$ 0.89-0.97 [9].

The Prowler and sprint trials were recorded with a hand held stop watch (100th second) and separated by a 2-3 minute rest period. The same researcher timed all of the trials. The trials were conducted on the team's grass turfed practice field (dry \& recently cut) and the participants wore their cleated footwear. The testing session was conducted in late November immediately following the football offseason. All of the participants had previous experience performing the Prowler sled push as well as sprint drills.

\subsection{Statistical Analysis}

The study used a repeated measures cross-over design with respect to the warmup strategies and the effects on the dependent variables. The dependent variables were compared between warmup conditions with a dependent t-test. Significance for these comparisons was set at $\alpha=0.05$ (one-tailed).

\section{Results}

Sixteen High School male football players participated in the study. The average age, height, and body mass of the participants is presented in Table 1 . All of the participants completed the study procedures without incident.

The average of the three Prowler push trials and sprint trials was used for analysis (Table 2). There was a significant improvement in the Prowler push times $(\approx 8.9 \%$ ) following the PAP warmup $P<0.01$. There was also a significant reduction in sprint times $(\approx 1.5 \%)$ following the PAP warmup strategy $P<0.01$.

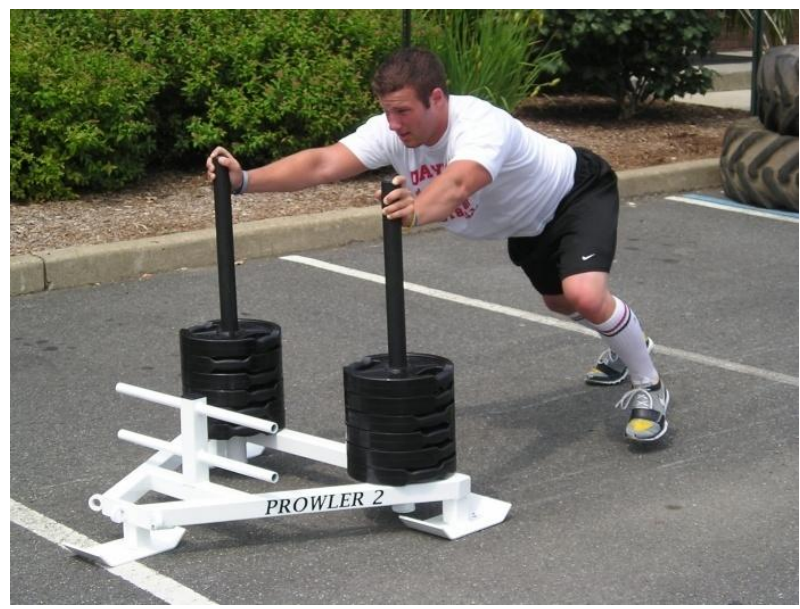

Fig. 1 Prowler resistance sled (total mass 91 kilograms pushed for 9.1 meters).

Table 1 Participant descriptive information.

\begin{tabular}{lll}
\hline Age (years) & Height $(\mathrm{cm})$ & Mass $(\mathrm{kg})$ \\
\hline $16.3 \pm 1.1$ & $177.2 \pm 6.7$ & $83.1 \pm 18.0$ \\
\hline
\end{tabular}

High School Male Football players, $N=16$ (mean \pm sd).

Table 2 Trial time results.

\begin{tabular}{llll}
\hline \multicolumn{2}{c}{ Sled Push } & \multicolumn{2}{c}{ Sprint } \\
\hline Dynamic & PAP & Dynamic & PAP \\
\hline $5.27 \pm 0.90$ & $4.80 \pm 0.67 *$ & $3.24 \pm 0.39$ & $3.19 \pm 0.39 *$ \\
\hline Prowler weighted sled push and sprint times in seconds (mean \pm \\
sd), $* P<0.01$.
\end{tabular}


On an individual basis, $87.5 \%$ of the participants improved the Prowler push times, $6.25 \%$ demonstrated no change and $6.25 \%$ with a slower time. With regards to the sprint times, $75 \%$ of the participants improved, $6.25 \%$ with no change, and $18.75 \%$ with slower times.

\section{Discussion}

The purpose of this study was to determine the effects of a PAP warm-up strategy that used a high intensity BSQ set as a potentiating exercise on weighted resistance sled pushes (Prowler) and subsequent short sprint times. The results of this study suggest that the BSQ PAP warmup strategy was on average effective at increasing Prowler performance $(\approx 8.9 \%)$. It was also determined that subsequent average 18.2 meter sprint times were reduced (1.5\%).

The improvement in the Prowler and 18.2 meter sprint times as a result of the BSQ PAP warmup found in this study is consistent with prior studies which have demonstrated that a PAP warmup can significantly increase both upper and lower body power output [10-15]. Fourteen of the participants experienced an improvement in sled push times while the others experienced no change or a slower time as the result of the PAP warmup. Twelve of the participants also experienced reduced 18.2 meter sprint times, while one had no change and three had slower times. While the reduction in sprint time was small, it is worth noting that the participants did not get slower following the PAP warmup and Prowler push trials.

The training status of an athlete is the primary factor necessary for a PAP training strategy to be successful [16-19]. Further, the National Strength and Conditioning Association (NSCA) suggests that PAP protocols should be "reserved for resistance-trained power athletes with high relative strength" [2]. The participants in this study were High School male American football players between the ages of 14-18 years of age with at least 6 months of resistance training experience which included BSQs. Likewise, all of the participants performed sprint drills and sled pushes as part of their typical strength and conditioning exercises. With that said, the participants in this study clearly did not meet the NSCA's PAP training status recommendations. Hence, prior training status and relative strength may have been partially responsible for some of the participants not experiencing any measureable potentiation as a result of the PAP BSQ protocol used in this study. In contrast, it appears that the training status and relative strength did not impede most of participants from experiencing a potentiating effect of the BSQ PAP. Albeit, the potentiating effect may have been even greater if the participants were more advanced in their resistance training status. Hence, the positive results of this study suggest that exploring PAP protocols in High School level athletes requires further investigation.

The success of a PAP protocol requires the determination of the optimal stimulus that allows for the coexistence (minimization) of fatigue while the muscles are in a potentiated state [20]. With that said, it is possible that the volume and/or intensity of the BSQ PAP warmup used in this study was too great, leading to a detrimental level of fatigue and hindering the performance of the sled pushes and sprint times. The BSQ PAP warmup volume used in this study could be easily modified by reducing the number of repetitions in the BSQ progression sets as well as reducing the culminating BSQ set to one or two repetitions at $85 \%$ of 1-RM.

Recovery time following the potentiating exercise has also been shown to be critical with regards to maximizing the outcomes of a PAP protocol where 3-12 minutes has been identified as ideal in well trained athletes $[16,18]$. The current study used a rest period of 4 minutes, which based on our observations was appropriate (the participants appeared rested and ready to get started with the Prowler sled pushes). However, we feel that the rest period to be employed in a PAP protocol should be developed on an individual basis considering the training status of the athlete as well as how the individual feels on a certain day (sleep, 
nutritional status, life stresses, etc.).

It has been suggested that the potentiated period following a potentiating stimulus is between 2-20 minutes in elite athletes $[16,19]$. In the current study, the sprint trials were conducted immediately following (2-3 minutes) the Prowler trials. Hence the total time frame that a potentiating affect could occur in this study was as follows: 4 minute rest period following the BSQ PAP, 2-3 minutes separating the three trials of Prowler pushes and sprint trials. Hence the participants completed all of the trials (Prowler and sprints) in a time frame ranging from 14-19 minutes. The results of this study indicated that there was a potentiating effect for the Prowler pushes as well as the sprints which occurred in the aforementioned time frame that is in agreement with that suggested by others $[16,19]$.

To explore the effectiveness of the BSQ PAP warmup, the average of three trials was used for statistical analysis. We felt that an average of multiple trials would likely represent the type of impact the PAP warmup might contribute to a game like situation where multiple plays occur. With that said, we also examined the best scores for the sled push and sprint times under both warmup conditions. The results indicated that the BSQ PAP warmup strategy was effective at decreasing Prowler push $(P<0.05)$ and 18.2 meter sprint times $(P<0.05)$, which agreed with the initial analysis using the average of the three trials.

The potentiating exercise selected for this study was the BSQ. We choose the BSQ for two primary reasons. Firstly, the BSQ has been used successfully in prior research as a potentiating exercise for increasing sprint speed [21]. Secondly, the BSQ is a closed chain, full body, multi-joint exercise known for its ability to place "controlled stress on the musculoskeletal system" [22]. In retrospect, it would be of interest to determine if using a deadlift (DL) could yield similar results. The DL uses similar muscle groups and posterior chain muscle recruitment patterns as the BSQ and would only require the use of an Olympic bar and weights, hence, eliminating the need for a squat rack.
As a point of interest, we also explored the relationship between the participants Prowler push and sprint times. A Pearson correlation coefficient PCC was used to compare sprint times with the Prowler times as corrected for participant body mass (Prowler push time/(sled mass/body mass). The rational for this correction being that a lower body mass individual would be Prowler pushing a higher percentage of body mass load than a heavier individual. The PCC between the sprint and corrected Prowler push times was $\mathrm{r}=$ 0.75 , which is considered high [23]. The coefficient of determination $\left(\mathrm{CD}=\mathrm{r}^{2}\right) \mathrm{CD}=0.56$ which indicates $56 \%$ of Prowler and sprint ability come from common factors [23]. In other words, a high percentage of physical attributes that contribute to the performance in Prowler push times also contribute to the performance in sprint times. Given that the Prowler push is a horizontal plane power output movement and that sprinting is a horizontal plane speed (velocity) movement, it is reasonable that a high relationship would exist as power is a function of velocity (power $=$ force $\times$ velocity) [22].

Coaches could use the information about the correlation between Prowler performance and sprint times to aid in developing strength and conditioning protocols. Specifically, if this correlation held true with a variety of athletes and lifters, it would suggest that improving either the Prowler or sprint times would improve the other at a proportional rate. In fact some research has been conducted that agrees with this notion where sled training has been used to improve sprinting ability $[4,5]$. This relationship could greatly benefit coaches and athletes wanting to improve either Prowler push or sprint performance, both being attributes directly related to football playing ability.

This study demonstrated that a BSQ PAP can acutely enhance Prowler push and sprint times. Coaches might consider implementing a similar BSQ PAP warmup on a regular basis in training as it reasonable to expect that there would be chronic improvements in both Prowler push and sprint ability. 
Finally, Coaches might also consider trying the PAP warmup used in this study to determine if an immediate result could be yielded on the playing field. Specifically, a team could use a PAP warmup prior to a game and then pay attention to the execution of those sprinting as well as those handling the blocking and/or rushing tasks. Receivers and running backs might be slightly faster, which can be the difference between making a break away play and/or potentially scoring. Likewise, passers or blockers controlling the line of scrimmage early can lead to big plays as well as setting the momentum for the game. While plays at the end of the game are often considered the most crucial, we contend that all plays count equally. Implementing a strategy that could help win the first 20 minutes of the game could set the atmosphere for the entire game. Likewise, could a second PAP warmup be conducted just prior to the second half starting?

\section{Conclusions}

Within the parameters of this study it is concluded that: using a PAP BSQ warmup is a viable way to improve resistive weighted sled pushes and subsequent sprint times; further, PAP protocols should not be reserved for advanced resistance-trained athletes with very high relative strength.

\section{Conflict Statement}

None of the authors have any known conflict of interest with the manufacturer of the Prowler or otherwise.

\section{References}

[1] Hodgson, M., Docherty, D., and Robbins, D. 2005. "Post-activation Potentiation." Sports Medicine 35 (7): 585-95.

[2] NSCA. 2016. NSCA Hot Topic: Post-Activation Potentiation (PAP). Accessed January 19, 2016. https://www.nsca.com/Education/Articles/Hot-Topic-Post -Activation-Potentiation-\%28PAP\%29/

[3] Bishop, D. 2008. "An Applied Research Model for the Sport Sciences.” Sports Medicine 38 (3): 253-63.

[4] Zafeiridis, A., Saraslanidis, P., Manou, V., Ioakimidis, P., Dipla, K., and Kellis, S. 2005. "The Effects of Resisted
Sled-Pulling Sprint Training on Acceleration and Maximum Speed Performance." Journal of Sports Medicine and Physical Fitness 45 (3): 284-90.

[5] Clark, K. P., Stearne, D. J., Walts, C. T., and Miller, A. D. 2010. "The Longitudinal Effects of Resisted Sprint Training Using Weighted Sleds vs. Weighted Vests." Journal of Strength and Conditioning Research 24 (12): 3287-95.

[6] Baechle, T. R., Earle, R. W., and Ratamess, N. A. 2008. Essentials of Strength and Training and Conditioning (3rd Edition). Champaign, IL: Human Kinetics, 350-451.

[7] Jenkins, N. M. D., and Palmer, T. 2012. "Implement Training for Concentric-Based Muscle Actions." Strength \& Conditioning Journal 34 (2): 1-7.

[8] Tano, G., Bishop, A., Climstein, M., and DeBeliso, M. 2016. "The Reliability of the Prowler in High School Male Football Players.” Journal of Sports Science 4 (4): 183-8.

[9] Miller, T. 2012. NSCA's Guide to Tests and Assessments. Champaign, IL: Human Kinetics.

[10] Harris, C., Dolny, D., Browder, K., Adams, K. J., and DeBeliso, M. 2004. "The Effect of Prior Contractile Activity on Power Output." Medicine \& Science in Sports \& Exercise 36 (5): S350.

[11] Harris, C., Kipp, K., Adams, K. J., DeBeliso, M., and Berning J. M. 2011. "The Effects of High Intensity Warm-up on Shot Put Performance." Medicine \& Science in Sports \& Exercise 43 (5): S600.

[12] Harris, C., Moore, J., DeBeliso, M., Adams, K. J., Berning, J., and Hansen, C. 2006. "The Effects of Heavy Squats on Potentiating 30 Second Wingate Test Performance." Medicine \& Science in Sports \& Exercise 38 (5): S292.

[13] Berning, J. M., Adams, K. J., DeBeliso, M., Sevene-Adams, P. G., Harris, C., and Stamford, B. A. 2010. "Effect of Functional Isometric Squats on Vertical Jump in Trained and Untrained Men." Journal of Strength and Conditioning Research 24 (9): 2285-9.

[14] Mallander, S., Berning, J., Pederson, S., Adams, K. J., DeBeliso, M., Stamford, B., and Maud, P. 2008. "5RM Bench Increases Medicine Ball Put Distance in Trained Males and Females." Medicine \& Science in Sports \& Exercise 40 (5): S262.

[15] Dove, B. C., Sevene, T. G., Harris, C., DeBeliso, M., Adams, K. J., Carson, C. A., and Berning, J. M. 2013. "5RM Bench Press Increases Medicine Ball Put Distance in Untrained Males But not Untrained Females." Medicine \& Science in Sports \& Exercise 45 (5): S597.

[16] Gullich, A., and Schmidtbleicher, D. 1996. "MVC-Induced Short-Term Potentiation of Explosive Force." New Studies in Athletics 11: 67-81.

[17] Hrysomallis, C., and Kidgell, D. 2001. "Effect of Heavy Dynamic Resistive Exercise on Acute Upper-Body Power." Journal of Strength and Conditioning Research 15 (4): 
426-30.

[18] Kilduff, L. P., Bevan, H. R., Kingsley, M. I., Owen, N. J., Bennett, M. A., Bunce, P. J., Hore, A., M., Maw, J. R., and Cunningham, D. J. 2007. "Postactivation Potentiation in Professional Rugby Players: Optimal Recovery." Journal of Strength and Conditioning Research 21 (4): 1134-8.

[19] Gilbert, G., and Lees, A. 2005. "Changes in the Force Development Characteristics of Muscle Following Repeated Maximum Force and Power Exercise." Ergonomics 48 (11-14): 1576-84.

[20] Rassier, D. E., and MacIntosh, B. R. 2000. "Coexistence of Potentiation and Fatigue in Skeletal Muscle." Brazilian
Journal of Medical and Biological Research 33 (5): 499-508.

[21] Yetter, M., and Moir, G. L. 2008. "The Acute Effects of Heavy Back and Front Squats on Speed during 40-m Sprint Trials." Journal of Strength and Conditioning Research 22 (1): 159-65.

[22] O'Shea, P. 2000. Quantum Strength Fitness II (Gaining the Winning Edge). Applied Strength Training \& Conditioning for Winning Performance. Corvallis, OR: Patrick's Books.

[23] Safrit, M. J., and Wood, T. M. 1995. Introduction to Measurement in Physical Education and Exercise (3rd Ed.) St. Louis, MO: Mosby. 